\title{
Hypoxia does not predict lack of benefit from adjuvant radiotherapy for patients with early stage breast cancer
}

\author{
(c) The Author(s), under exclusive licence to Springer Nature Limited 2022
}

There is a need to identify patients with early stage breast cancer for whom radiotherapy and its associated toxicities might be avoided. Tutzauer et al. hypothesised that a hypoxia biomarker might be used but found that adjuvant radiotherapy following surgery reduces risk of recurrence irrespective of hypoxia status.

British Journal of Cancer (2022) 126:1111-1112; https://doi.org/ 10.1038/s41416-022-01707-8

Hypoxia drives tumour progression, and is associated with resistance to radiation and many chemotherapeutic agents. All solid tumours have some areas where oxygen levels fall below physiological normoxia, and patients with the most hypoxic tumours have a poorer prognosis. As a lack of oxygen causes radioresistance, Tutzauer et al. hypothesised that patients with hypoxic tumours would derive no benefit from postoperative radiotherapy when used as part of breast conserving treatments [1].

Interestingly, I would not formulate such an hypothesis because patients with the most versus least hypoxic tumours have a poorer prognosis irrespective of treatment by surgery or radiotherapy. My opinion draws on knowledge of the work of Hockel and colleagues in carcinoma of the cervix, many years of research developing and validating hypoxia-associated gene signatures and published studies investigating the prognostic significance of hypoxia-associated markers.

Radiation researchers explored methods for measuring tumour hypoxia over the last 60 years with the first oxygen electrode studies reported in the 1960s [2]. Our ability to measure tumour oxygenation improved in the 1990s with the development of the Eppendorf $\mathrm{pO}_{2}$ histograph. The equipment used fine needle polarographic needle electrodes and an automatic stepper motor, which meant multiple oxygen tension measurements were made while avoiding tissue compression and bleeding artefacts associated with older style electrodes. A key finding of Eppendorf studies was published in 1996 when Hockel et al. showed that patients with the most hypoxic carcinomas of the cervix had decreased probabilities of disease-free and overall survival [3]. Importantly, tumour hypoxia increased locoregional failures with/without distant metastases, irrespective of whether surgery or radiation was the primary treatment.

Regarding development of hypoxia-associated gene signatures, an advantage is classifiers can be tested in both surgical and radiotherapy cohorts. There are now many publically available gene expression datasets, which are dominated by surgical treatment as exemplified by The Cancer Genome Atlas repository. The hypoxia-associated gene signatures developed by my group and collaborators were validated in both surgical and radiotherapy cohorts, i.e., the signatures are prognostic irrespective of whether surgery or radiation was the primary treatment. There are also plenty of studies where high expression of hypoxia markers (e.g. HIF-1a and (AIX) associated with a poor prognosis following surgery or radiotherapy.

While not agreeing with the hypothesis, the questions addressed by Tutzauer et al. are interesting and worthwhile. Also, the work involves a large cohort of patients with early stage disease who were randomised to receive breast-conserving surgery alone or with adjuvant whole breast radiotherapy randomised trials are arguably the only way to test whether a biomarker is predictive (benefit from a specific intervention) rather than just prognostic. The Swedish trial recruited patients between 1991 and 1997. Although the radiotherapy approach would be considered out-dated, that does not matter when the endpoints relate to tumour recurrence (ipsilateral breast recurrence, any breast cancer recurrence, breast cancer death). The randomised trial cohort provides an excellent resource to identify biomarkers that predict benefit from adjuvant radiotherapy for patients with early stage breast cancer, and the authors already derived such a classifier [4]. It was good to see the group tested the Torres-Roca 10-gene radiosensitivity index (RSI) signature in their 2019 publication. RSI did not validate in the Swedish trial, but it is well validated and has been shown to predict benefit from adjuvant radiotherapy. A signature reflecting radiosensitivity (radiotherapy specific) rather than hypoxia (poor prognosis irrespective of treatment modality) should be a better predictor of benefit from adjuvant radiotherapy.

In their latest study, the authors addressed their hypothesis by measuring HIF-1a protein expression (in 985 of 1178 randomised patients). Of course, HIF-1a does not measure tumour oxygenation but, as the master regulator of cellular adaptation to hypoxia, reflects downstream effects. HIF-1 stabilisation occurs around $1-2 \%$ oxygen, but also occurs due to oncogenic alterations found in tumours that reflect an aggressive (more likely to recur) phenotype. Oxygen sensitises cells to the effects of sparsely ionising radiation. The half-maximal enhancement occurs at oxygen levels of $\sim 0.5 \%$ so radiosensitivity is only slightly reduced at $1 \%$. However, while measuring HIF-1a might not be the best way of assessing hypoxia, it is of interest to address the question of whether there is any interaction between HIF-1a expression and 
1112

benefit from adjuvant radiotherapy. Although many studies found high HIF-1a associates with a poor cancer prognosis, some in early stage disease treated with surgery (head and neck, upper gastrointestinal), found the opposite. Tutzauer et al. provide high-level evidence (findings from a randomised trial) that HIF-1a positivity is an adverse prognostic factor in early stage breast cancer and there is no interaction with use of adjuvant radiotherapy. The authors also show for the first time that HIF-1a positivity associates with ipsilateral breast recurrence. A particularly interesting finding was that most tumour samples from surgically removed ipsilateral recurrences had the same HIF1a status as their corresponding primary tumour, indicating that hypoxia and HIF-1a positivity is an inherent tumour trait.

Tutzauer et al. also assessed hypoxia using published gene signatures extracted from GeneChip Human Exon 1.0 ST microarray data (922 patients). They showed many were prognostic and correlated with HIF-1 a status, i.e., they reflect transcriptional HIF-1 activity. The authors also found that patients benefited from adjuvant radiotherapy regardless of hypoxia scores.

Where next? Further evaluation of hypoxia towards its inclusion in molecular profiling would be better using a gene signature rather than immunohistochemistry. Platforms for measuring gene signatures are sensitive and reproducible for clinical use-more so than obtainable with a single protein and immunohistochemistry as illustrated by efforts to standardise Ki67 scoring across laboratories [5]. Therefore, further study of hypoxia signatures is warranted. The next aspect to consider is how to target hypoxia therapeutically. Perhaps a bioreductive or HIF-1 a specific agent? It would also be worth considering a simple option of having patients with the most hypoxic tumours breathe an oxygen enriched atmosphere (carbogen), which was shown to improve overall survival in patients with muscle-invasive bladder cancer [6].

\author{
Catharine M. L. West $\mathbb{D}^{1 凶}$ \\ ${ }^{1}$ Division of Cancer Sciences, The University of Manchester, Christie \\ Hospital NHS Foundation Trust, Wilmslow Road, Withington, \\ Manchester M20 4BX, UK. 『email: Catharine.west@manchester.ac.uk
}

\section{REFERENCES}

1. Tutzauer J, Sjöström M, Holmberg E, Karlsson P, Killander F, Leeb-Lundberg LMF et al. Breast cancer hypoxia in relation to prognosis and benefit from radiotherapy after breast-conserving surgery in a large, randomised trial with long-term follow-up. Br J Cancer. 2022. (in this issue)
2. Kolstad P. Oxygen tension and radiocurability in cancer of the cervix. Acta Obstet Gynecol Scand. 1965;43:100-2.

3. Hockel M, Schlenger K, Aral B, Mitze M, Schaffer U, Vaupel P. Association between tumor hypoxia and malignant progression in advanced cancer of the uterine cervix. Cancer Res. 1996;56:4509-15.

4. Sjostrom M, Chang SL, Fishbane N, Davicioni E, Zhao SG, Hartman L, et al. Clinicogenomic radiotherapy classifier predicting the need for intensified locoregional treatment after breast-conserving surgery for early-stage breast cancer. J Clin Oncol. 2019;37:3340-9.

5. Polley MY, Leung SC, Gao D, Mastropasqua MG, Zabaglo LA, Bartlett JM, et al. An international study to increase concordance in Ki67 scoring. Mod Pathol. 2015;28:778-86

6. Hoskin PJ, Rojas AM, Bentzen SM, Saunders MI. Radiotherapy with concurrent carbogen and nicotinamide in bladder carcinoma. J Clin Oncol. 2010;28: 4912-8.

\section{ACKNOWLEDGEMENTS}

The author is supported by the Manchester NIHR Biomedical Research Centre.

\section{AUTHOR CONTRIBUTIONS}

All contributions were from the single author.

\section{ETHICS APPROVAL AND CONSENT TO PARTICIPATE}

Not applicable.

\section{CONSENT TO PUBLISH}

Not applicable.

\section{COMPETING INTERESTS}

The author set up ManTRa Diagnostics in 2021.

\section{ADDITIONAL INFORMATION}

Correspondence and requests for materials should be addressed to Catharine M. L. West.

Reprints and permission information is available at http://www.nature.com/ reprints

Publisher's note Springer Nature remains neutral with regard to jurisdictional claims in published maps and institutional affiliations. 